Anestesiología

Enero-Marzo 2021

Vol. 44. No. 1. pp 13-21

doi: $10.35366 / 97773$

\section{Descripción de costos del tratamiento analgésico protocolizado con catéter epidural versus la analgesia convencional para la nefrectomía abierta del donador renal en el Instituto Mexicano del Seguro Social}

\author{
Cost description of protocolized analgesic treatment with epidural \\ catheter versus conventional analgesia for open kidney donor \\ nephrectomy in the Mexican Institute of Social Security
}

\author{
Dra. Dulce M Rascón-Martínez,* Dra. Martha Y Flores-Rodríguez, ${ }^{\ddagger}$ \\ Dra. Felipa L Hernández-Hernández,* Dra. Gilda Martínez-Castillo, ${ }^{\ddagger}$ \\ Dr. Gerardo A Caballero-Souza, ${ }^{\S}$ Dra. Roceli Arrocena-Salgado," \\ Dr. Antonio Castellanos-Olivares ${ }^{\pi}$
}

Citar como: Rascón-Martínez DM, Flores-Rodríguez MY, Hernández-Hernández FL, Martínez-Castillo G, CaballeroSouza GA, Arrocena-Salgado R, y col. Descripción de costos del tratamiento analgésico protocolizado con catéter epidural versus la analgesia convencional para la nefrectomía abierta del donador renal en el Instituto Mexicano del Seguro Social. Rev Mex Anest. 2021; 44 (1): 13-21. https://dx.doi.org/10.35366/97773

RESUMEN. Introducción: El dolor posterior a la nefrectomía abierta en donadores renales trasciende en la recuperación plena de las actividades de la vida diaria y expone un impacto económico en las instituciones de salud. Objetivo: Describir el costo de la analgesia protocolizada durante el período postoperatorio inmediato del paciente donante renal. Material y métodos: Realizamos un ensayo clínico controlado, aleatorizado, no ciego que comparó dos tratamientos analgésicos diferentes: uno protocolizado versus la analgesia convencional mediante un modelo de análisis de costos desde la perspectiva institucional. Resultados: En promedio, la analgesia protocolizada obtuvo un costo mayor con 53.25 US\$ (52.34-53.79) versus el tratamiento analgésico convencional con 18.84 US\$ (4.55-19.34), U = -6.0, $p<$ 0.001 . Sin embargo, $89.2 \%$ de los pacientes con analgesia protocolizada calificó como excelente esta intervención a diferencia de $41.2 \%$ de los pacientes que recibieron el tratamiento analgésico convencional, $\chi^{2}=18.78 ; p<0.001$. Del mismo modo, los pacientes con analgesia protocolizada estuvieron más satisfechos reportando una mediana: $(\mathrm{Me})=$ 10; $($ Percentil25 = 10 - Percentil75 = 10) en comparación con el otro tratamiento: Me = 8; (Percentil25 = 8 - Percentil75 = 9) $(U=-5.9, p<0.001)$. Conclusiones: En pacientes sometidos a nefrectomía abierta para donación renal, el uso de analgesia protocolizada con catéter epidural demostró una diferencia clínica y estadísticamente significativa con respecto a los reportes de dolor, eficacia y un mayor grado de satisfacción, aunque no disminuyó los costos de la atención analgésica durante el postoperatorio inmediato.

ABSTRACT. Introduction: Pain in renal donors after open nephrectomy, transcends in full recovery of activities in daily life and exposes an economic impact in health institutions. Objective: To describe the costs of protocolized analgesia during the immediate postoperative period of renal donor patient. Material and methods: We conducted a longitudinal, randomized, non-blinded clinical trial which compared two different analgesic treatments: one protocolized versus conventional analgesia through a cost analysis model from the Institutional Social Security perspective. Results: In average, protocolized analgesia obtained a higher cost with 53.25 US\$ (52.34-53.79) opposed to the conventional analgesia treatment with 18.84 US\$ (4.55-19.34); $U=-6.0, p<0.001$. However, $89.2 \%$ of the patients in protocolized analgesia qualified this intervention as excellent compared to the conventional treatment with only $41.2 \%, \chi^{2}=18.78 ; p<0.001$. Similarly, patients in protocolized analgesia were more satisfied with a reported median $(\mathrm{Me})=10 ;(25$ th percentile $=10-75$ th percentile $=10)$ compared to those who received conventional treatment, $M e=8$; $(25$ th percentile $=8-75$ th percentile $=9)(U=-5.9, p<0.001)$. Conclusions. The use of Protocolized Analgesia with epidural catheter showed a clinical and statistically significant difference concerning to pain, efficacy and a greater satisfaction report in patients undergoing open nephrectomy for renal donation, although does not decrease the costs of analgesic care during immediate postoperative period.

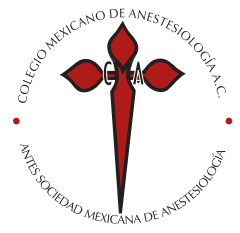

Palabras clave:

Costos, analgesia protocolizada, donador renal.

Keywords:

Cost, protocolized analgesia, renal donor.

* Maestría en Ciencias de la Salud. Departamento de Anestesiología. UMAE Hospital de Especialidades «Dr. Bernardo Sepúlveda G» del Centro Médico Nacional Siglo XXI, Instituto Mexicano del Seguro Social.

‡ Departamento de Anestesiología. UMAE Hospital de Especialidades «Dr. Bernardo Sepúlveda G» del Centro Médico Nacional Siglo XXI, Instituto Mexicano del Seguro Social. $\S$ Director Ciencia de Datos BBVA Bancomer. Maestría en Ciencias-Estadística.

" Maestría en Administración de Hospitales. Departamento de Anestesiología. UMAE Hospital de Especialidades «Dr. Bernardo Sepúlveda G» del Centro Médico Nacional Siglo XXI, Instituto Mexicano del Seguro Social. ^ Maestría en Ciencias Médicas, Maestría en Administración de Organizaciones de la Salud. Jefe del Departamento de Anestesiología. UMAE Hospital de Especialidades «Dr. Bernardo Sepúlveda G» del Centro Médico Nacional Siglo XXI, Instituto Mexicano del Seguro Social.

Correspondencia:

Dulce M Rascón-Martínez Departamento de Anestesiología. UMAE Hospital de Especialidades «Dr. Bernardo Sepúlveda G» del Centro Médico Nacional Siglo XXI, Instituto Mexicano del Seguro Social, Avenida Cuauhtémoc Núm. 330, Col. Centro, Alcaldía Cuauhtémoc, Ciudad de México, México. Tel. 56276900, ext. 21607. E-mail: dradulcerascon@gmail.com

Recibido para publicación: 14-11-2018 Aceptado para publicación: 02-04-2019 


\section{INTRODUCCIÓN}

$\mathrm{E}$ trasplante renal es el tratamiento de elección en los pacientes con enfermedad renal crónica en estadío final ${ }^{(1)}$. En países como en los Estados Unidos de América se tiene la tasa más alta de donación renal (24 donantes por millón de habitantes). Según la United Network for Organ Sharing/The Organ Procurement and Transplantation Network (UNOS/OPT) $)^{(2,3)}$ tan sólo en el año 2007 en países miembros de Eurotransplant, como Austria, Bélgica y Alemania, se han reportado tasas de trasplantes renales de 4 a 7.5 riñones provenientes de donadores vivos por millón de habitantes.

En la actualidad, 20\% de todos los trasplantes renales en Eurotransplant y $40 \%$ en EUA se realizan con donantes vivos $^{(4,5)}$. El trasplante de donante vivo se ha considerado una necesidad lamentable debido al éxito de este tipo de trasplante (según lo valorado por la supervivencia de los injertos y los pacientes) y la escasez de órganos de donantes fallecidos ${ }^{(6)}$, por lo que el acto de la donación se torna complejo, ya que depende de muchos factores e interacciones. En este sentido, el abordaje elegido para la obtención de riñones de donantes vivos, dígase el abordaje transperitoneal clásico, el extraperitoneal sub- o supracostal y/o el laparoscópico transperitoneal o retroperitoneal, dependerá del paciente, la experiencia del cirujano y lógicamente de los recursos hospitalarios ${ }^{(7,8)}$. A excepción del abordaje laparoscópico, todos los abordajes mencionados son sumamente invasivos y dolorosos, lo que nos lleva a hablar acerca de la seguridad del donante, en la que se ha reportado una tasa histórica de mortalidad de $0.03 \%$ con la nefrectomía abierta, tasa que no se ha modificado con la introducción de la nefrectomía laparoscópica de donante vivo $^{(9)}$.

Por otro lado, las medidas relativas a necesidades de analgésicos, intensidad de dolor, estancia hospitalaria y tiempo necesario hasta la reincorporación al trabajo son significativamente menores bajo un procedimiento laparoscópico, aunque para su implementación se requieren de recursos adicionales. No obstante, la estancia hospitalaria más breve y la reincorporación más rápida al trabajo pueden compensar los mayores costes iniciales ${ }^{(10)}$.

En México, la nefrectomía abierta sigue siendo el abordaje más frecuente en la mayoría de los centros en los que se realiza la cirugía de trasplante renal, lo cual puede resultar en dolor persistente después de la cirugía. Un estudio alemán reportó una incidencia de dolor persistente postquirúrgico después de la donación renal en 34\% de los donadores a los 22 meses (rango: 9-57 meses) después de la nefrectomía, y en 55\% de esos casos el dolor interfirió con las actividades de la vida diaria ${ }^{(11)}$. En consecuencia, se han reportado repercusiones económicas por el inapropiado manejo del dolor postquirúrgico. Otro estudio encontró que el costo promedio por paciente fue de 1,869.00 dólares por admisiones o readmisiones asociadas a dolor y 986.00 dólares por visitas al Servicio de Urgencias ${ }^{(12)}$. Lo anterior está explicado por la cronificación del dolor agudo cuando se emplea una mala terapéutica analgésica y se le denomina dolor postquirúrgico persistente. La definición clásica es la de un dolor que está presente por tres o más meses posterior al evento quirúrgico y se ha reportado una incidencia estimada de 10 a $50 \%{ }^{(13,14)}$. Por ello, la planeación preoperatoria óptima y la implementación de protocolos analgésicos se vuelve necesaria e imprescindible para poder así minimizar la práctica de tratamientos analgésicos convencionales que sólo promueven: a) la inadecuada evaluación del dolor y la falta de monitorización del tratamiento durante las primeras 24 a 72 horas en las cuales el dolor se considera mayor, b) la falta de comunicación entre los servicios tratantes, c) la ausencia de protocolos de analgesia y pobre adherencia a los mismos cuando existen, d) la deficiencia en los programas de educación y en la estandarización del conocimiento en profesionales de la salud, e) el uso deficiente de técnicas analgésicas efectivas de acuerdo a procedimientos específicos y a la evidencia científica, f) la no optimización de los recursos propios del hospital, g) la documentación inadecuada del nivel del dolor en el expediente clínico y, finalmente, $\mathrm{h}$ ) el retraso del inicio de la analgesia por diversas actividades del personal de enfermería. Para nuestra tranquilidad existen ya una gran variedad de lineamientos analgésicos que se encuentran ampliamente descritos y disponibles online para procedimientos quirúrgicos específicos ${ }^{(15-17)}$ y la analgesia del paciente donador renal sometido a nefrectomía abierta no es la excepción ${ }^{(18-20)}$.

En una revisión de Cochrane se concluyó que, para todos los tipos de cirugía, la analgesia epidural proporciona un mejor control del dolor postoperatorio cuando se comparó esta técnica con la administración intravenosa de opioides, incluyendo la técnica de analgesia controlada por el paciente. (Nivel de evidencia I) ${ }^{(21)}$. Jones y colaboradores publicaron un estudio en el que incluyeron a 42 pacientes que fueron sometidos a nefrectomía para donación renal bajo la colocación de un catéter epidural y el promedio de días de estancia hospitalaria resultó de 3.4 (rango: dos a ocho días) y la media por cargo de hospitalización fue de 15.169 dólares (rango: 10.733-29.579 dólares). Los autores concluyeron que la donación renal en vida desde el punto de vista de los donadores irrumpió sus vidas de forma mínima ${ }^{(22)}$.

Por lo tanto, cuando seleccionamos una estrategia analgésica, debemos considerar además de la eficacia y la efectividad, el costo ${ }^{(23)}$. Las consecuencias de planear o descontinuar un tratamiento llega a ser de interés cuando se comparan algunas de estas estrategias y ponen en valor de juicio si el costo adicional resultante es razonable ${ }^{(23)}$ y si se supera en eficacia a los manejos «convencionales» de analgesia, ya que éstos, habitualmente, consisten en el uso de múltiples medicaciones o la combinación de ellas, lo que potencializa el riesgo de 
sinergismos farmacológicos peligrosos. En la actualidad, estos esquemas «convencionales» son a menudo como insuficientes para mitigar el dolor severo, ya que se usa frecuentemente la leyenda «Por razón necesaria», lo que resulta en un control analgésico ineficaz. El objetivo final del estudio pretende evaluar el costo de la actividad analgésica protocolizada desde el punto de vista institucional usando un catéter epidural y hacer la comparación con la analgesia convencional en el período postquirúrgico del donante renal.

\section{MATERIAL Y MÉTODOS}

El estudio fue aprobado por los Comités de Ética y de Investigación del Hospital de Especialidades «Dr. Bernardo Sepúlveda G» del Centro Médico Nacional Siglo XXI del Instituto Mexicano del Seguro Social. La Dra. Dulce María Rascón Martínez fungió como investigador principal. Se tuvieron como variables independientes: a) esquema analgésico protocolizado con catéter epidural y b) esquema analgésico convencional por la Unidad de Trasplantes.

\section{Participantes}

Los sujetos fueron reclutados en el mismo hospital y fueron invitados a participar durante la visita preanestésica previa al evento quirúrgico durante el año 2018 y 2019. Se incluyeron pacientes catalogados como ASA 1 y 2 (hipertensión controlada con uno o dos antihipertensivos) según la clasificación de la American Society of Anaesthesiologists y las guías clínicas para trasplante renal y donador vivo ${ }^{(24)}$, programados para cirugía de nefrectomía mayores de 18 años, ambos géneros y que pudieron ser evaluados después de su intervención. Excluimos a los pacientes que a juicio del médico anestesiólogo y/o investigadores no fueron candidatos a colocación de catéter epidural o situaciones que justificaran la interrupción de las maniobras que comprometieran las variables de seguridad del estudio y que no se haya tenido el tiempo suficiente para evaluar el resultado final.

\section{Diseño del estudio y procedimientos}

Se realizó un estudio de costos usando un modelo de ensayo clínico controlado, aleatorizado, ciego simple. Usamos este modelo, ya que así exploramos variables de resultado secundarias relacionadas a la analgesia y la satisfacción. Una vez que se contó con el consentimiento informado y la evaluación clínica basal se realizó una aleatorización y con el mecanismo de la tómbola los participantes fueron asignados para recibir: a) analgesia protocolizada durante 48 horas con ropivacaína vía catéter epidural al $0.125 \%^{(25)}+$ metamizol 1 g IV cada 8 horas + paracetamol 1 g vía oral cada seis horas, a cargo del Servicio de Medicina del Dolor del hospital, o bien; b) tratamiento analgésico habitual o convencional de acuerdo a la indicación de los médicos tratantes en la Unidad de Trasplante Renal. Este último constó del uso de uno o más antiinflamatorios no esteroideos tipo ketorolaco, metamizol y clonixinato de lisina, alternados y/o combinados. En este mismo grupo se empleó infiltración de la herida quirúrgica y se podían indicar o no opioides tipo tramadol o buprenorfina como analgesia de rescate a consideración de los médicos del Servicio de Trasplantes.

Para identificar a los participantes, los investigadores asignaron un código de identificación y las evaluaciones de dolor fueron realizadas a las 24 y 48 horas posteriores al evento quirúrgico. Para el análisis de costo se registró el total de las medicaciones analgésicas empleadas, costo de las medicaciones coadyuvantes para el manejo de los efectos adversos y el costo del equipo de infusión elastomérica Baxter ${ }^{\circledR}$ $\mathrm{LV} 5$, flujo alto a $5 \mathrm{~mL} / \mathrm{h}$, volumen $275 \mathrm{~mL}$. Adicionalmente se consideraron los días y el costo de la hospitalización hasta su egreso. El horizonte de análisis se llevó a cabo durante dos días y desde la perspectiva del proveedor de servicios, es decir, el Instituto Mexicano del Seguro Social. Se incluyeron únicamente costos directos inmediatos en moneda nacional y consecuencias inmediatas (náuseas, vómito, incidentes con la bomba epidural, interconsultas a especialista para manejo del dolor, manejo terapéutico por punción accidental de duramadre, etcétera. Las actividades sanitarias se consideraron según el Acuerdo ACDO. AS3.HCT.271119/329.P.DF y sus anex os, dictado por el H. Consejo Técnico, relativo a la aprobación de los Costos Unitarios por Nivel de Atención Médica que rigieron para el ejercicio del año 2020 y el Diario Oficial de la Federación (primera sección) el 30 de diciembre del 2019 y el sistema electrónico de compras gubernamentales ${ }^{(26)}$.

\section{Análisis estadístico}

El tamaño de la muestra se seleccionó con base al diseño del estudio y a la diferencia de medias de los datos presentados en el artículo publicado por el Dr. Thomas G. Peters y colegas, Living kidney donation: recovery and return to activities or daily living. Clin Transplantation 2000: 14: 433-438(23). Se tomaron en cuenta los días de estancia intrahospitalaria en pacientes sometidos a nefrectomía abierta bajo anestesia general y analgesia epidural. Este estudio reportó una media de estancia hospitalaria de 3.4 días \pm 1.197 . De acuerdo con esta desviación y usando un poder $\beta$ de $80 \%$ con un error $\alpha$ de $5 \%$, el número resultante por grupo fue de 29 pacientes, deliberadamente incrementamos a 36 pacientes considerando $20 \%$ de las posibles pérdidas. Las variables cuantitativas se describieron utilizando medidas de tendencia central y de dispersión, de acuerdo con el tipo de distribución de los datos. Las variables cualitativas se expresaron en frecuencias absolutas y/o porcentajes. Para las asociaciones entre las variables 
cualitativas se utilizó la prueba de $\chi^{2}$ y para las cuantitativas la prueba de U de Mann-Whitney, o T de Student en caso de que siguieran una distribución normal. Se hizo un análisis de dos colas con un nivel de significancia de 0.05 usando el paquete estadístico SPSS versión 21 para MAC.

\section{RESULTADOS}

Se contemplaron en total 86 pacientes, el análisis estadístico finalmente incluyó a 71 sujetos, de los cuales fueron excluidos $12.9 \%(n=15)$. El esquema general del diseño del estudio y los motivos de exclusión se describen a detalle en la Figura 1. Del total de la muestra analizada $53.5 \%(n=38)$ de los pacientes correspondieron al género femenino y $46.5 \%$ ( $n=$ 33) al género masculino. La edad promedio de los sujetos fue de $38.29 \pm 11.85$ años. La Tabla 1 muestra por grupos las variables clínico-demográficas.

\section{Analgesia}

El dolor fue medido mediante la técnica clinimétrica, escala visual numérica (EVN). Dado que el dolor es subjetivo, para el análisis estadístico, los pacientes fueron catalogados con escalas tipo Likert en: sin dolor (0 puntos), dolor leve (1-3 puntos), moderado (4-7 puntos) y aquéllos con dolor severo (> 8 puntos). Las evaluaciones se llevaron a cabo en dos momentos diferentes: el primer y segundo día posterior a la intervención quirúrgica. El clonixinato de lisina fue el analgésico no esteroideo usado con más frecuencia con $75.7 \%$ $(\mathrm{n}=28)$ en el grupo de analgesia protocolizada y 100\% ( $\mathrm{n}$ $=34$ ) en el grupo de con analgesia habitual, seguido por el metamizol $21.6 \%(\mathrm{n}=8)$ y finalmente el ketorolaco $2.7 \%$ $(\mathrm{n}=1) ; \chi^{2}=9.471, \mathrm{p}=0.009$. Los analgésicos tipo opioides fueron utilizados en $57.7 \%(n=41)$ de los pacientes, $22 \%$ ( $n$ = 9) en el grupo de analgesia protocolizada con catéter epidural y 78\% ( $n=32)$ en pacientes con tratamiento analgésico habitual. En ambos grupos, el anestésico local más utilizado fue la ropivacaína por vía epidural para la analgesia protocolizada y la modalidad de infiltración de la herida quirúrgica por el cirujano se utilizó en 30.2\% $(n=16)$ de los pacientes con tratamiento analgésico convencional.

Adicionalmente se registró la cantidad de dolor durante el reposo y al movimiento, así como la demanda de rescates analgésicos, durante las primeras 48 horas. La Tabla 2, muestra con detalle el comportamiento de estas variables.

\section{Eventos adversos, calidad del sueño y recuperación}

La escala tipo Likert también fue usada para catalogar la severidad de los efectos adversos durante las primeras 48 horas del postoperatorio. El análisis no encontró diferencias significativas entre los grupos, de igual forma no hubo repor-

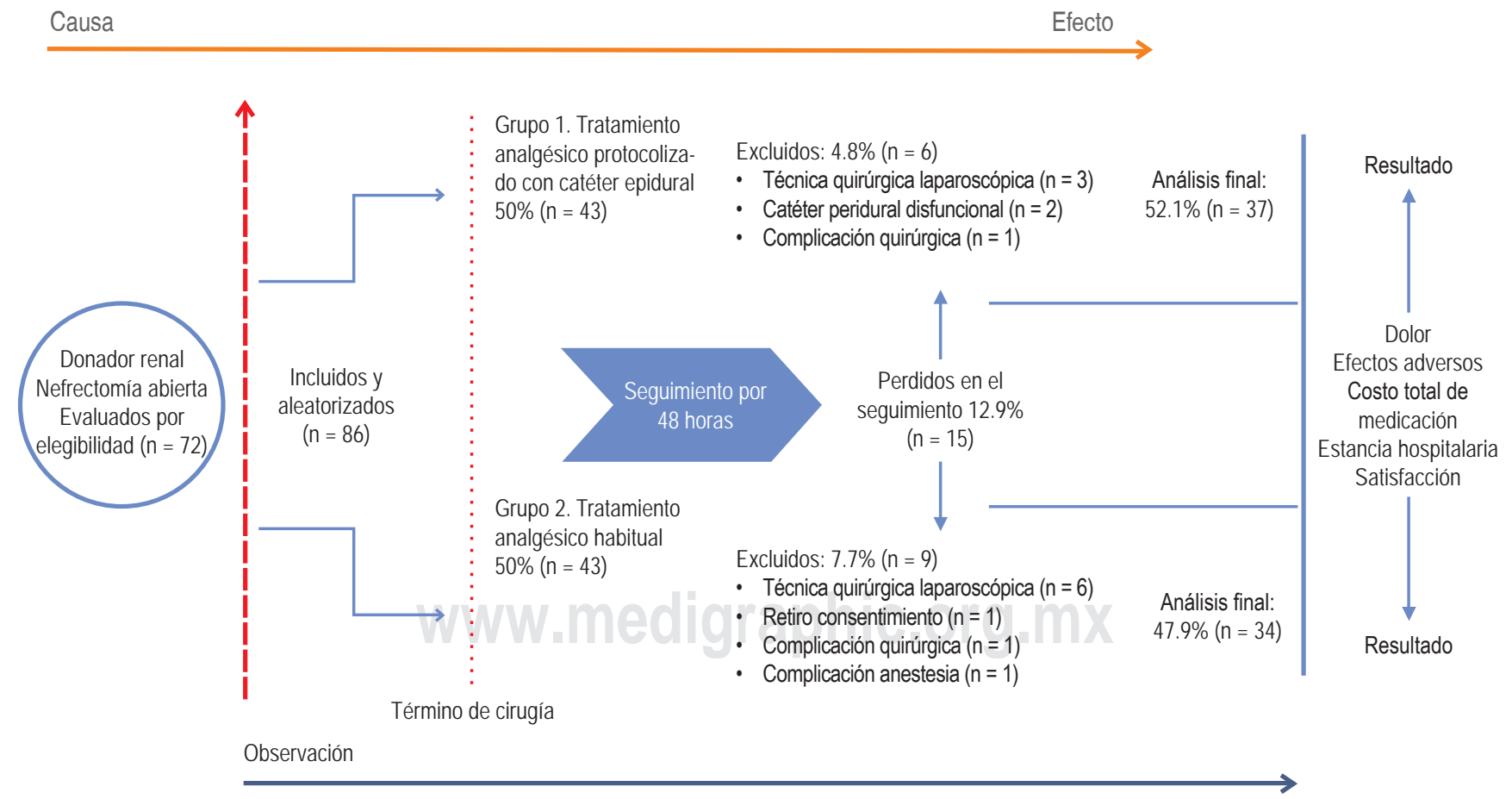

Figura 1: Flujograma de CONSORT. Diseño del estudio que incluyó el seguimiento de pacientes sometidos a nefrectomía para donación renal con el fin de comparar los costos y efectos de las dos alternativas analgésicas para el manejo del dolor agudo postoperatorio por 48 horas. 
Tabla 1: Variables clínico-demográficas de la población en estudio.

\begin{tabular}{|c|c|c|c|}
\hline & Analgesia protocolizada $n=37$ & Analgesia habitual $n=34$ & Estadística \\
\hline Género n (\%) & & & $\chi^{2}=0.146, p=0.702$ \\
\hline Femenino & $19(26.80)$ & $19(26.80)$ & \\
\hline Masculino & $18(25.40)$ & $15(21.10)$ & \\
\hline Edad en años (media $\pm \mathrm{DE}$ ) & $37.97( \pm 11.77)$ & $38.62( \pm 11.93)$ & $t=0.22, p=0.133$ \\
\hline IMC (media $\pm \mathrm{DE})$ & $25.4( \pm 2.55)$ & $25.7( \pm 3.13)$ & $t=0.44, p=0.656$ \\
\hline ASA $n(\%)$ & & & $\chi^{2}=8.82, p<0.001^{*}$ \\
\hline ASA 1 & $18(25.40)$ & $28(39.40)$ & \\
\hline ASA 2 & $19(26.80)$ & $6(8.50)$ & \\
\hline \multicolumn{4}{|l|}{ PAM (mmHg) (media \pm DE) } \\
\hline Día 1 & $81.3(9.44)$ & $84.5(10.57)$ & $t=1.13, p=0.178$ \\
\hline Día 2 & $81.5(7.13)$ & $81.94(7.91)$ & $t=1.60, p=0.835$ \\
\hline Opioide n (\%) & & & $\chi^{2}=33.996, p<0.001^{*}$ \\
\hline Tramadol & $0(0.00)$ & $24(75.00)$ & \\
\hline Morfina & $3(33.30)$ & $2(6.30)$ & \\
\hline Buprenorfina & $0(0.00)$ & $6(18.80)$ & \\
\hline Fentanilo & $6(66.70)$ & $0(0.00)$ & \\
\hline
\end{tabular}

IMC = índice de masa corporal, DE: desviación estándar, ASA: clasificación del estado físico de la American Society of Anesthesiologists, PAM: presión arterial media, mmHg: milímetros de mercurio, $\chi^{2}=$ Chi cuadrada, $\mathrm{t}=\mathrm{T}$-Student,${ }^{*}$ Significancia estadística.

tes de efectos secundarios de intensidad moderada a severa y tampoco se reportaron datos de parestesia y/o estreñimiento en ambos grupos. La retención urinaria y la necesidad de colocar una sonda urinaria se presentó en $1.4 \%(\mathrm{n}=1)$ de los pacientes del grupo analgesia protocolizada con catéter epidural.

Con respecto a la recuperación posterior a la cirugía, tomamos en cuenta el inicio de las siguientes actividades para el grupo de analgesia protocolizada versus el grupo con analgesia convencional, por la distribución de los datos se presentan en mediana (Me) y rango intercuartílico (RIC), respectivamente. La deambulación registró una $\mathrm{Me}=24$ (1) vs 24 (5) horas, $\mathrm{p}=0.683$; la vía oral: $\mathrm{Me}=21$ (5) vs 19 (6) horas, $\mathrm{p}=0.108$; la diuresis: $\mathrm{Me}=20$ (4) vs 20 (6) horas, $\mathrm{p}$ $=0.655 ; \mathrm{y}$ finalmente, el inicio de las evacuaciones: $\mathrm{Me}=30$ (2) vs 25.5 (6) horas, $p \leq 0.001$. La distribución porcentual de los eventos adversos se muestra en la Tabla 3.

\section{Costos de la intervención analgésica}

El resultado de estas variables se expresó en medianas (Me) y percentiles (25-75). Se adoptó como unidad monetaria el dólar americano (US\$) con una equivalencia de 19 pesos mexicanos para comparar los resultados con estudios internacionales.

Para ambos grupos el consumo de analgésicos por los días totales de estancia hospitalaria resultó en: Me $=18.61$ US\$ (17.75-19.29), los costos totales por estancia hospitalaria: Me $=1,203.89$ US $\$(1,203.89-1,203.89)$, el costo de medicaciones analgésicas y equipo utilizado: $\mathrm{Me}=52.26$ US $\$$ (18.84-53.39). El costo total de la atención analgésica se sumó al costo total de la estancia hospitalaria resultando: $\mathrm{Me}=1,255.94$ US\$ (1,222.73-1,257.42). Para calcular el costo de los esquemas terapéuticos fueron utilizados los costos directos de los analgésicos (costo unitario), materiales descartables (jeringas, agujas, diluyentes), dispositivos tipo infusor elastomérico, equipos de bloqueo epidural, venoclisis, anestésicos locales, medicación para el manejo de efectos adversos secundarios a la intervención analgésica, expresada por evento referido en este ensayo clínico. Excluimos los costos que no incidieron directamente sobre la actividad analgésica (Tabla 4).

\section{Perspectiva del paciente}

Antes del egreso de la Unidad de Trasplantes formulamos dos preguntas a los pacientes: ¿qué tan eficaz considera usted que fue el tratamiento analgésico brindado? En el primer cuestionamiento $89.2 \%(n=33)$ de los pacientes con analgesia protocolizada calificaron como excelente ese tratamiento analgésico y $10.8 \%(n=4)$ lo calificó como bueno, lo que se comportó diferente con los pacientes con analgesia habitual/ convencional, en el que $41.2 \%(n=14)$ la calificaron como excelente, $47.1 \%(n=16)$ como buena y $11.8 \%(n=4)$ la consideraron entre mala y regular $\left(\chi^{2}=18.78 ; \mathrm{p} \leq 0.001\right)$.

Finalmente, con respecto al segundo cuestionamiento que evaluó la satisfacción del derechohabiente, los pacientes con analgesia protocolizada arrojaron mejores resultados en ese sentido: $\mathrm{Me}=10$; (P25: 10-P75: 10) en comparación con los que recibieron analgesia convencional: $\mathrm{Me}=8$; (P25: 8-P75: 9) $(\mathrm{U}=-5.9, \mathrm{p} \leq 0.001)$, ello acorde a una escala análoga numérica de 0 al 10 . 


\section{DISCUSIÓN}

La donación de órganos durante la vida es un acto de gran altruismo, con impactos familiares, sociales, económicos y psicológicos únicos que se suman a la edad en la que se realiza. Solís y colaboradores observaron un promedio de edad del paciente donador renal de $36 \pm 10$ años, muy similar a lo percibido en nuestro estudio y con una población de características sociodemográficas similares ${ }^{(27)}$.

La estrategia de tratamiento más común para el alivio del dolor postoperatorio posterior a la nefrectomía de donador renal en los centros hospitalarios es multimodal, utilizando fármacos analgésicos antiinflamatorios no esteroideos y opioides; sin embargo, la mayor parte de los antiinflamatorios no esteroideos son nefrotóxicos, la infiltración de anestésico local no alivia el dolor en los tejidos profundos y los opioides tienen efectos adversos incluyendo prurito, náuseas, vómitos, depresión respiratoria y disminución de la motilidad gastrointestinal. Los estudios publicados por Forastiere, Panaro, Hosgood y Meltiem y colegas coinciden en el beneficio clínico de los anestésicos locales con distintas alternativas de administración. Por lo tanto, el uso de una técnica analgésica neuroaxial puede conducir a una mejor calidad de recuperación postquirúrgica ${ }^{(28,29)}$.

En este sentido, el implementar un protocolo analgésico fue útil, con un impacto positivo en los donantes de riñón. Acorde con nuestros resultados, el tratamiento analgésico con infusión continua de anestésico local mediante la colocación de un catéter epidural a nivel de T11-T12 o bien T12-L1, a lo largo de 48 horas, disminuyó los reportes de dolor en reposo y movimiento, así como la demanda de analgésicos de rescate en los primeros dos días posteriores a la intervención quirúrgica. Nuestros resultados son similares a lo reportado por Oliveira y su equipo, quienes encontraron que $60 \%$ de

Tabla 2: Reporte de dolor de los grupos analizados ajustado a escalas tipo Likert.

\begin{tabular}{|c|c|c|c|}
\hline & $\begin{array}{l}\text { Analgesia protocolizada } \\
\qquad n=37 n(\%)\end{array}$ & $\begin{array}{c}\text { Analgesia habitual } \\
n=34 \mathrm{n}(\%)\end{array}$ & Estadística \\
\hline \multicolumn{4}{|l|}{ Dolor } \\
\hline \multicolumn{4}{|l|}{ Día 1} \\
\hline Reposo & & & $\chi^{2}=1.38, p=0.708$ \\
\hline Ausente & $6(16.2)$ & $5(14.7)$ & \\
\hline Leve & $13(35.1)$ & $10(29.4)$ & \\
\hline Moderado & $17(45.9)$ & $16(47.1)$ & \\
\hline Severo & $1(2.7)$ & $3(8.8)$ & \\
\hline Movimiento & & & $\chi^{2}=2.49, p=0.476$ \\
\hline Ausente & $1(2.7)$ & $1(2.9)$ & \\
\hline Leve & $13(35.1)$ & $7(20.6)$ & \\
\hline Moderado & $19(51.4)$ & $19(55.9)$ & \\
\hline Severo & $4(10.8)$ & $7(20.6)$ & \\
\hline Rescates analgesia & & & $\chi^{2}=10.02, p=0.018^{*}$ \\
\hline 0 & $22(59.5)$ & $8(23.5)$ & \\
\hline 1 & $12(32.4)$ & $21(61.8)$ & \\
\hline 2 & $3(8.1)$ & $4(11.8)$ & \\
\hline 3 & $0(0.0)$ & $1(2.9)$ & \\
\hline \multicolumn{4}{|l|}{ Día 2} \\
\hline Reposo & & & $\chi^{2}=11.61, p=0.003^{*}$ \\
\hline Ausente & $22(59.5)$ & $7(20.6)$ & \\
\hline Leve & $14(37.8)$ & $23(67.6)$ & \\
\hline Moderado & $1(2.7)$ & $4(11.8)$ & \\
\hline Severo & $0(0.0)$ & $0(0.0)$ & \\
\hline Movimiento & & & $\chi^{2}=15.73, p<0.001^{*}$ \\
\hline Ausente & $10(27.0)$ & $1(2.9)$ & \\
\hline Leve & $23(62.2)$ & $19(55.9)$ & \\
\hline Moderado & $3(8.1)$ & $14(41.2)$ & \\
\hline Severo & $1(2.7)$ & $0(0.0)$ & \\
\hline Rescates analgesia & & & $\chi^{2}=16.71, p<0.001^{*}$ \\
\hline 0 & 34 (91.9) & $18(52.9)$ & \\
\hline 1 & $2(5.4)$ & $16(47.1)$ & \\
\hline 2 & $1(2.7)$ & $0(0.0)$ & \\
\hline
\end{tabular}

$\chi^{2}=$ Chi cuadrada, ${ }^{*}$ Significancia estadística. 
Tabla 3: Sintomatología de efectos adversos ajustado a la Escala tipo Likert en los grupos analizados.

\begin{tabular}{|c|c|c|c|c|c|c|}
\hline & \multicolumn{2}{|c|}{$\begin{array}{c}\text { Analgesia protocolizada } \\
n=37 n(\%)\end{array}$} & \multicolumn{2}{|c|}{$\begin{array}{c}\text { Analgesia habitual } \\
n=34 n(\%)\end{array}$} & \multicolumn{2}{|c|}{ Estadística } \\
\hline & Día 1 & Día 2 & Día 1 & Día 2 & Día 1 & Día 2 \\
\hline Náusea & & & & & $\chi^{2}=1.29, p=0.255$ & $\chi^{2}=0.55, p=0.456$ \\
\hline Ausente & 33 (89.2) & 33 (89.2) & $27(79.4)$ & $32(91.5)$ & & \\
\hline Leve & $4(10.8)$ & $4(10.8)$ & $7(20.6)$ & $2(5.9)$ & & \\
\hline Vómito & & & & & $\chi^{2}=0.93, p=0.334$ & $\chi^{2}=0.93, p=0.334$ \\
\hline Ausente & $36(97.3)$ & $36(97.3)$ & $34(100.0)$ & $34(100.0)$ & & \\
\hline Leve & $1(2.7)$ & $1(2.7)^{\prime}$ & $0(0.0)$ & $0(0.0)$ & & \\
\hline Mareo & & & & & $\chi^{2}=0.26, p=0.606$ & $\chi^{2}=0.93, p=0.334$ \\
\hline Ausente & $35(94.6)$ & $36(97.3)$ & $33(97.1)$ & $34(100.0)$ & & \\
\hline Leve & $2(5.4)$ & $1(2.7)$ & $1(2.9)$ & $0(0.0)$ & & \\
\hline Sedación & & & & & $\chi^{2}=0.00, p=0.952$ & $\chi^{2}=1, p=1.000$ \\
\hline Ausente & $36(97.3)$ & $37(0.0)$ & $33(97.1)$ & $34(100.0)$ & & \\
\hline Leve & $1(2.7)$ & $0(0.0)$ & $1(2.7)^{\prime}$ & $0(0.0)$ & & \\
\hline Prurito & & & & & $\chi^{2}=1.10, p=0.294$ & $\chi^{2}=1, p=1.000$ \\
\hline Ausente & $37(100.0)$ & $37(100.0)$ & $33(97.1)$ & $34(100.0)$ & & \\
\hline Leve & $0(0.0)$ & $0(0.0)$ & $1(2.9)$ & $0(0.0)$ & & \\
\hline Dolor/calidad del sueño & & & & & $\chi^{2}=11.11, p=0.011^{*}$ & $\chi^{2}=13.77, p<0.001^{*}$ \\
\hline Ausente & $17(45.9)$ & $26(70.3)$ & $7(20.6)$ & $10(29.4)$ & & \\
\hline Leve & $14(37.8)$ & $10(27)$ & $17(50)$ & $24(70.6)$ & & \\
\hline Moderado & $3(8.1)$ & $1(2.7)$ & $10(29.4)$ & $0(0.0)$ & & \\
\hline Severo & $3(8.1)$ & $0(0.0)$ & $0(0.0)$ & $0(0.0)$ & & \\
\hline
\end{tabular}

$\chi^{2}=$ Chi cuadrada, ${ }^{*}$ Significancia estadística.

\begin{tabular}{|c|c|c|c|}
\hline & $\begin{array}{l}\text { Analgesia protocolizada } \\
\mathrm{n}=37, \mathrm{Me}(\mathrm{P} 25-\mathrm{P} 75)\end{array}$ & $\begin{array}{l}\text { Analgesia convencional } \\
\mathrm{n}=34, \mathrm{Me}(\mathrm{P} 25-\mathrm{P} 75)\end{array}$ & Estadística \\
\hline Consumo de analgésicos & $\$ 19.06(18.15-19.41)$ & $\$ 18.39(4.55-19.29)$ & $U=-2.3, p=0.020^{*}$ \\
\hline Costos totales del tratamiento analgésico & $\$ 53.25(52.34-53.79)$ & $\$ 18.84(4.55-19.34)$ & $U=-6.0, p<0.001^{*}$ \\
\hline Atención en analgesia y estancia hospitalaria & $\$ 1,246.63(1,246.65-1,248.44)$ & $\$ 1,212.21(1,207.39-1,212.71)$ & $U=-5.1, p<0.001^{*}$ \\
\hline Tiempo de estancia hospitalaria (días) & $3(3-3)$ & $3(3-3)$ & $U=-0.96, p=0.334$ \\
\hline
\end{tabular}

$\mathrm{Me}=$ mediana, $\mathrm{p}=$ percentil, $\mathrm{U}=\mathrm{U}$ de Mann-Whitney, ${ }^{*}$ Significancia estadistica.

30 donadores renales estuvieron satisfechos y 93.3\% estaban dispuestos a repetir la experiencia de la donación de riñón mediante una técnica analgésica con la colocación de un catéter epidural, el resto (6.7\%) usó analgesia controlada por el mismo paciente. En ese mismo estudio los aspectos positivos percibidos por los pacientes fueron: un control más adecuado del dolor postoperatorio, mayor información, atención y confianza en el equipo médico. Con respecto a eventos adversos, Oliviera reportó los siguientes efectos adversos: prurito (40\%), náuseas o vómitos (16.7\%), estreñimiento (6.7\%) y retención urinaria (3.3\%). Ningún donante mostró un estado alterado de conciencia, bloqueo motor o parestesias $^{(30)}$. Nuestro estudio no encontró diferencias con respecto a la recuperación, tampoco en los efectos adversos, a excepción por el inicio de las evacuaciones, que tomaron más tiempo en presentarse en el grupo de analgesia protocolizada. Es posible que tanto pacientes como cuidadores tengan mayor temor cuando se porta un catéter epidural y con ello, la restricción del movimiento afecte la motilidad intestinal. Por otro lado, observamos un mayor descanso nocturno con la técnica protocolizada, dado que hubo una menor interferencia del dolor con el sueño lo que pudo influir en los reportes de mayor satisfacción y eficacia según la percepción de los pacientes en ese mismo grupo.

La Donor Nephrectomy Outcomes Research (DONOR) Network, en Canadá, evaluó las consecuencias económicas de la donación renal e incluyó los costos de la medicación para el manejo del dolor reportando una media de 40 dólares por paciente ${ }^{(31)}$. Del mismo modo, en EUA el Kidney Donor Outcomes Cohort (KDOC) Study arrojó una media pondera- 
da de 77 dólares para la medicación, aunque en este último estudio no está claro si el costo fue exclusivamente para el manejo de analgesia o incluyó costos de antibióticos u otras medicaciones ${ }^{(32)}$. Además, tampoco se detalla qué tipo de esquemas o protocolos analgésicos fueron utilizados en ambos estudios. Estas cifras son similares a lo reportado en nuestro estudio (52.26 US) cuando comparamos únicamente el costo promedio en medicaciones para ambos grupos. No obstante, Klarenbach y Rodrigue y colegas publicaron sus resultados para los años 2014 y 2016, respectivamente. Por lo que estas cifras pueden variar si nos situamos en el año actual.

Por otro lado, algunas publicaciones que evalúan costos señalan que existe la tendencia de no hacer las mediciones pertinentes para demostrar un beneficio, al menos monetario $^{(33)}$. En nuestro estudio, la intervención analgésica protocolizada obtuvo un mayor costo comparada con el tratamiento analgésico convencional al considerar que la estancia hospitalaria fue similar en ambos grupos. Sin embargo, y acorde con la percepción de los pacientes, la eficacia y el grado de satisfacción, se comportaron mejor en el grupo de analgesia protocolizada con catéter epidural. Consideramos que, aunque los costos sean mayores, éstos deben ser aceptables por el aspecto bioético que rodea a estos pacientes que realizan un acto auténticamente altruista, ya que el donante vivo no obtiene un beneficio físico directo, por lo que se espera que las consecuencias de la cirugía y los efectos psicosociales que la misma genera sean lo más beneficiosos posibles ${ }^{(34)}$.

Retomando el punto anterior, una de las limitantes del estudio es su descripción parcial, dado que, el objetivo mayor fue ofrecer una descripción de costos en analgesia expresada en términos monetarios con la evaluación de una sola dimensión: el beneficio ${ }^{(35)}$. La descripción de costos se caracteriza porque no compara cursos alternativos de acción y su principal propósito es el reporte de costos asociados a una determinada intervención, por lo que no se realizó un análisis de costo incremental, la incertidumbre del costo y de consecuencias, ya que no se trató de un análisis de costo-utilidad (ACU), o de costo efectividad (ACE), mismos que tienen un enfoque multidimensional que considera: los años de vida ajustados por calidad (QALYs), los años de vida ajustados por discapacidad (DALYs) y consecuencias de una intervención ${ }^{(36)}$. Adicionalmente, los costos presentados en esta descripción pueden no ser representativos de otras instituciones y/o programas de trasplante en nuestro país.

Dado lo anterior, los autores sugerimos que nuestros resultados sean interpretados con cautela; no obstante, este proyecto es de relevancia clínica, ya que podría ofrecer las bases para realizar en un futuro un análisis económico en salud más complejo en este contexto. Los resultados informan acerca del potencial que tiene la planeación y protocolización de los esquemas de analgesia, que atiendan a una mayor atención percibida por el paciente durante el acto de donación y a una convalecencia más amigable.

Los autores sugerimos la optimización en la calidad de la atención analgésica como un derecho humano fundamental y como un principio ético de bienestar en pacientes en quienes la donación en vida debe considerarse un regalo de valor extraordinario y no debemos exponerlos a sufrimiento innecesario; por tanto, los costos que se generen para lograr ese objetivo han de sopesarse desde el punto de vista de una pequeña inversión.

\section{CONCLUSIONES}

El uso de analgesia protocolizada con catéter epidural demostró mejor comportamiento con respecto a los reportes de dolor en reposo, movimiento y requerimiento de rescates analgésicos, así como mayor grado de eficacia y satisfacción acorde a la percepción de los pacientes, aunque no disminuye los costos de la atención analgésica durante la hospitalización en comparación al grupo que recibió tratamiento analgésico convencional.

\section{REFERENCIAS}

1. Wolfe RA, Ashby VB, Milford EL, Ojo AO, Ettenger RE, Agodoa LY, et al. Comparison of mortality in all patient on dialysis, patients on dialysis awaiting transplantation, and recipients of a first cadaveric transplant. N Engl J Med. 1999;341:1725-1730.

2. United Network for Organ Sharing. Available in: http://www.unos.org/.

3. The Organ Procurement and Transplantation Network. Available in: http://www.optn.org.

4. Oosterlee A, Rahmel A, van Zwet W (eds). Annual report 2005. Eurotransplant International Foundation, Leiden: 2005.

5. Malaise J, Van Deynse D, Dumont V, Lecomte C, Mourad M, Dufrane $\mathrm{D}$, et al. Non-heart-beating donor, 10-year experience in a Belgian transplant center. Transplant Proc. 2007;39:2578-2579.

6. Sells RA, Johnson R, Hutchinson I. Recommendations on the use of living kidney donors in the United Kingdom. British Transplantation Society. Br Med J (Clin Res Ed). 1986;293:257-258.
7. Dalla Valle R, Mazzoni MP, Capocasale E, Busi N, Pietrabissa A, Moretto C, Gualtierotti M, Massa M, Mosca F, Sianesi M. Laparoscopic donor nephrectomy: short learning curve. Transplant Proc. 2006;38:1001-1002.

8. Berardinelli L. Technical problems in living donor transplantation. Transplant Proc. 2005;37:2449-2450.

9. Matas AJ, Bartlett ST, Leichtman AB, Delmonico FL. Morbidity and mortality after living kidney donation, 1999-2001: survey of united states transplant centers. Am J Transplant. 2003;3:830-834.

10. Hadjianastassious VG, Johnson RJ, Rudge CJ, Mamode N. 2509 living donor nephrectomies, morbidity and mortality, including the UK introduction of laparoscopic donor surgery. Am J Transplant. 2007;7:2532-2537.

11. Gottschalk A, Götz J, Zenz M, Schmerz. Pain and quality of life for living donors after nephrectomy. Schmerz (Berlin, Germany) [Schmerz], ISSN: 1432-2129, 2009;23:502-509; Publisher: Springer-Verlag; PMID: 1976363. 
12. Coley KC, Williams BA, DaPos SV, Chen C, Smith RB. Retrospective evaluation of unanticipated admissions and readmissions after day surgery and associated cost. J Clin Anesth. 2002;14:349-353.

13. Steyaert A, De Kock M. Chronic postsurgical pain. Curr Opin Anaesthesiol. 2012;25:584-588.

14. Johansen A, Romundstad L, Nielsen CS, Schirmer H, Stubhaug A. Persistent postsurgical pain in a general population: prevalence and predictors in the Tromso study. Pain. 2012;153:1390-1396.

15. Kehlet H, Wilkinson RC, Fischer HB, Camu F. PROSPECT: evidencebased, procedure-specific postoperative pain management. Best Pract Res Clin Anaesthesiol. 2007;21:149-159.

16. http://www.anzca.edu.au/resources/college-publications.

17. Warfield CA, Kahn CH. Acute pain management. Programs in U.S. hospitals and experiences and attitudes among U.S. adults. Anesthesiology. 1995;83:1090-1094.

18. Forastiere E, Sofra M, Giannarelli D, Fabrizi L, Simone G. Effectiveness of continuous wound infusion of $0.5 \%$ ropivacaine by On-Q pain relief system for postoperative pain management after open nephrectomy. $\mathrm{Br}$ J Anaesth. 2008;101:841-847.

19. Kehlet H, Dahl J. Anaesthesia, surgery, and challenges in postoperative recovery. Lancet. 2003;362:1291.

20. Panaro F, Gheza F, Piardi T, Woehl Jaegle ML, Audet M, Cantù M, et al Continuous infusion of local anesthesia after living donor nephrectomy: a comparative analysis. Transplant Proc. 2011;43:985-987.

21. Werawatganon T, Charuluxanun S. Patient controlled intravenous opioid analgesia versus continuous epidural analgesia for pain after intra-abdominal surgery. Cochrane Database Syst Rev. 2005;(1):CD004088.

22. Jones KW, Peters TG, Charlton RK, Lenz BJ, Walker GW, Repper $\mathrm{S}$, et al. Current issues in living donor nephrectomy. Clin Transplant. 1997;11:505-510.

23. Peters TG, Repper SM, Jones KW, Walker GW, Vincent M, Hunter RD. Living kidney donation: recovery and return to activities of daily living. Clin Transplant. 2000;14:433-438.

24. Guidelines for living donor kidney transplantation. Fourth Edition January 2018. British Transplantation Society. Disponible online en: www.bts.org.uk.
25. Patil SS, Kudalkar AG, Tendolkar BA. Comparison of continuous epidural infusion of $0.125 \%$ ropivacaine with $1 \mu \mathrm{g} / \mathrm{mL}$ fentanyl versus $0.125 \%$ bupivacaine with $1 \mu \mathrm{g} / \mathrm{mL}$ fentanyl for postoperative analgesia in major abdominal surgery. J Anaesthesiol Clin Pharmacol. 2018;34:29-34.

26. Diario Oficial de la Federación. Instituto Mexicano del Seguro Social. Disponible en: https://dof.gob.mx/nota_detalle.php?codigo=5583111\& fecha=30/12/2019\&print=true.

27. Solis E, Evangelista LA. Epidemiological characteristics of the largest kidney transplant program in Mexico: Western National Medical Center, Mexican Institute of Social Security. Transplant Proc. 2016;48:1999-2005.

28. Güner Can M, Göz R, Berber İ, Kaspar Ç, Çakır Ü. Ultrasound/ laparoscopic camera-guided transversus abdominis plane block for renal transplant donors: a randomized controlled trial. Ann Transplant. 2015;20:418-423.

29. Hosgood S, Thiyagarajan U. Randomized clinical trial of transversus abdominis plane block versus placebo control in live-donor nephrectomy. Transplantation. 2012;94:520-525.

30. Oliveira B, Mascarenhas C. Assessment of the degree of satisfaction among living kidney donors. Transplantation Proceedings. 2011;43:43-47.

31. Klarenbach S, Gill S. Economic consequences incurred by living kidney donors: a Canadian multi-center prospective study. Am J Transplant. 2014;14:916-922.

32. Rodrigue J, Schold J. For the kdoc study group. Direct and indirect costs following living kidney donation: findings from the KDOC Study. Am J Transplant. 2016;16:869-887.

33. Bartha E, Carlsson P, Kalman S. Evaluation of costs and effects of epidural analgesia and patient-controlled intravenous analgesia after major abdominal surgery. Br J Anaesth. 2006;96:111-111.

34. De Graaf Olson W, Bogett-Dumlao A. Living donors' perception of their quality of health after donation. Prog Transplant. 2001;11:108-115.

35. Drummond MF, O’Brien B, Stoddart GL, Torrance GW. Methods for the economic evaluation of health care programs. 2nd ed., Oxford: Oxford University Press; 1997.

36. Zarate V. Evaluaciones económicas en salud: conceptos básicos y clasificación. Rev Med Chile. 2010;138:93-97. 